\title{
Método para Seleção de Softwares de Apoio ao Processo Ensino-Aprendizagem de Disciplinas de Programação
}

\author{
Rafael Santos ${ }^{1}$, Leonardo Gonçalves ${ }^{1}$, Benilda Silva ${ }^{2}$, Carlos Portela ${ }^{1}$, Fabricio \\ Farias $^{1}$ \\ ${ }^{1}$ Faculdade de Sistemas de Informação - Universidade Federal do Pará (UFPA) \\ Cametá - PA - Brasil \\ ${ }^{2}$ Faculdade de Educação - UFPA - Cametá - PA - Brasil \\ \{morimrafael, leo.widgeon16\}@gmail.com, benildaveloso@hotmail.com, \\ $\{$ csp, fabriciosf $\}$ aufpa.br
}

\begin{abstract}
This paper presents a method for the selection of different pieces of software to support the teaching and learning process on programming disciplines. In order to validate this method, a survey considering different pieces of software available at Google Play store was carried out. During the survey, five teaching and learning applications, covering the course of "Computer Programming I" were selected. These applications were subjected to both ergonomic and pedagogical analyzes, which were carried out by professionals in the information technology field. At the end, a ranking was prepared, according to the proposed method, being selected software "Learn Java" and "Java Tutorial - offline".
\end{abstract}

Resumo. Este trabalho apresenta um método para seleção de softwares de apoio ao ensino e aprendizagem de disciplinas de Programação. Para validação do método proposto foi realizado um levantamento de softwares disponibilizados na loja virtual Google Play, onde foram selecionados cinco aplicativos voltados ao ensino-aprendizagem da disciplina de Programação de Computadores I. Estes foram submetidos às análises ergonômicas $e$ pedagógicas realizadas por profissionais da área de tecnologia da informação. Ao final, um ranking foi elaborado, conforme o método proposto, sendo selecionados os softwares Learn Java e Java Tutorial - offline.

\section{Introdução}

Os computadores e dispositivos móveis estão cada vez mais presentes como ferramentas mediadoras no âmbito do processo de ensino-aprendizagem [Lima et al. 2016]. O aumento no uso dessas ferramentas proporciona a criação de ambientes escolares complexos, mais tecnológicos e interativos para todos os agentes envolvidos no referido processo, isto é, alunos e professores [Carlet e Czyzewski 2016]. Nestes ambientes, cada vez mais se faz o uso de novos métodos de ensino-aprendizagem baseados na interação com softwares educativos que, por sua vez, visam apresentar aos usuários diversos conteúdos de forma organizada para um aprendizado progressivo.

Para Nunes et al. (2017), o crescente acesso as tecnologias digitais de informação e comunicação através da Internet e dispositivos móveis proporciona a 
experiência de modelos pedagógicos inovadores que podem apoiar o processo de ensino-aprendizagem. Nesta perspectiva, Twining et al. (2013) destacam que os professores em formação devem, essencialmente, conhecer diferentes ferramentas de apoio ao ensino e aprendizagem, como forma de integração inovadora da tecnologia para atender os estudantes do século 21. Já Martínez-Rodriguez et al. (2019) constatam que tal aplicação de novos métodos de ensino e aprendizagem podem impactar positivamente na confiança dos estudantes e em seus hábitos de estudo fora da sala de aula, tornando, assim, válida a aplicação de alternativas que garantam o bem-estar dos agentes envolvidos no processo de ensino-aprendizagem.

No contexto dos cursos de computação, tanto os professores quanto os alunos da área da tecnologia da informação utilizam softwares como ferramentas de apoio para a superação de diversas dificuldades enfrentadas durante a vida acadêmica. Em relação às dificuldades enfrentadas, Oliveira et al. (2017) destacam que na Universidade Federal do Pará (UFPA) os maiores índices de reprovações estão presentes em disciplinas que envolvem as áreas de algoritmos e programação de computadores. Como solução, os autores propuseram um software para auxilio no ensino-aprendizagem de algoritmos, mas, disponibilizaram a solução gratuitamente na loja do Google Play somente até 2020. Já os resultados obtidos por Silveira e Alcântara (2014) relatam o grande número de desistências dos alunos da área de tecnologia da informação e justificam os índices, associando-os a diversos problemas como: a complexidade dos assuntos abordados ou ausência de novos estímulos a partir da aplicação do modelo tradicional de ensino. Assim, os autores destacam a necessidade da busca por novas alternativas que possam vir a reduzir os índices apresentados.

De acordo com Oliveira et al. (2017) e Silveira e Alcântara (2014), cenários compostos por elevados números de reprovações em algoritmos e programação, assim como compostos por grande número de desistências, demonstram a real necessidade de se buscar, urgentemente, novas soluções que possam funcionar como ferramentas de suporte dentro ou fora da sala de aula.

Diante deste contexto, este trabalho objetiva especificar um método para seleção de softwares de apoio ao ensino-aprendizagem de alunos que cursam disciplinas de programação. Para validar o método, foi realizado um estudo de caso a partir da seleção de cinco aplicativos relacionados à disciplina de Programação de Computadores I, que é uma das disciplinas com maiores índices de reprovação no curso de Sistemas de Informação da UFPA Campus Cametá [Viana et al. 2019]. Para validação dos softwares selecionados, foram convidados especialistas da área de tecnologia da informação para utilização e validação de forma qualitativa dos softwares.

O restante deste trabalho está organizado da seguinte forma: na Seção 2 é apresentado o referencial teórico. A Seção 3 descreve o método que foi utilizado durante a pesquisa. A Seção 4 apresenta o estudo de caso realizado para validação da pesquisa. A Seção 5 apresenta os resultados obtidos. Por fim, a Seção 6 apresenta as considerações finais e as próximas etapas da pesquisa.

\section{Referencial Teórico}

No âmbito educacional, Nunes e Santos (2018) destacam que o uso de softwares voltados para o ensino-aprendizagem dentro da sala de aula pode servir como auxílio 
para os alunos, visto que ajuda eles na fixação e no melhor aproveitamento do conteúdo que foi repassado durante a aula. Deste modo, o relacionamento da educação com a tecnologia enriquece a qualidade do processo de ensino-aprendizagem, o que causa impacto direto nos métodos, nas práticas e nos recursos do ensino. Assim, os alunos ganham um ambiente alternativo para que sejam postos em prática os novos conhecimentos adquiridos, da mesma maneira que podem ter um melhor controle sobre o ritmo de seu aprendizado.

A adoção de ambientes alternativos também é impulsionada pelo crescimento do número de usuários de dispositivos móveis. Segundo o Instituto Datafolha (2014) 43 milhões de brasileiros acessam a Internet por celular ou tablet. Acompanhando o crescimento do acesso aos dispositivos móveis, também cresce o número de softwares, na modalidade de aplicativo, voltados para a área educacional. Com isso há uma grande diversidade desses softwares, no entanto, muitos não conseguem suprir a necessidade básica de construção do conhecimento; ou sequer apresentam fins pedagógicos [Gouvêa e Nakamoto 2015]. Com isso, observa-se uma dificuldade na etapa de escolha da ferramenta adequada para a utilização no ensino-aprendizagem de determinado conteúdo.

\subsection{Ensino-Aprendizagem de Programação}

Nos cursos da área da computação, as disciplinas com base em algoritmos e programação são as que mais provocam desistências de alunos. Tal constatação pode ser validada de forma estatística por dois estudos. O primeiro realizado pelo Instituto Federal Fluminense e o segundo pela Universidade Federal do Pará. No primeiro, foi constatado que o índice de reprovação semestral nas disciplinas de algoritmos pode alcançar uma média semestral de 35,66\% [Barcelos et al. 2009]. Já no segundo, os indicadores revelam que durante o período de 2011 a 2018 cerca de 52,73\% das reprovações que aconteceram nas disciplinas do curso de Sistemas de Informação possuem como base teórica a disciplina de algoritmos [Viana et al. 2019], existindo, assim, uma dificuldade latente de aprendizagem que precisa ser combatida através da aplicação de novas alternativas e métodos de ensino e aprendizagem.

Em relação as dificuldades latentes, um estudo conduzido por Bittencourt et al. (2013) destaca que este problema está ligado ao fato de se ter um curto período de tempo para aprendizagem e fixação de diversos conceitos específicos sobre pensamento computacional e abstração. Deste modo, havendo a necessidade da apresentação de soluções que permitam um contato mais qualitativo entre os alunos e os conteúdos abordados em sala de aula.

De acordo com Oliveira, Bitencourt e Monteiro (2016) a introdução de aplicativos para auxiliar o aprendizado dos alunos torna esse aprendizado mais abrangente, interativo e agradável, pois além do espaço da sala de aula eles podem expandir a zona de aprendizagem para qualquer lugar, assim como passam a ter um ambiente composto por novos elementos. No entanto, tal adesão de tecnologias de apoio gera o desafio de como escolher o software que mais se adequa ao contexto de sua aplicação educacional. Por essa razão, é necessário investigar e verificar um método viável para escolha do aplicativo que poderá ser adotado por professores e alunos, dentro e fora do ambiente escolar. 


\subsection{Seleção e Avaliação de Ferramentas}

Não se deve avaliar um software de apoio ao ensino-aprendizagem apenas com base em características de qualidade técnicas, mas também em características relacionadas ao processo educacional que dizem respeito a aspectos pedagógicos. Neste sentido, Barbosa e Costa (2017) propuseram uma técnica de seleção e avaliação de ferramentas para o ensino de pensamento computacional, levando em consideração diferentes aspectos técnicos e pedagógicos. Para isso, os autores propuseram uma metodologia baseada nas etapas de busca, seleção e avaliação das ferramentas. Já Costa et al. (2015) investigaram uma metodologia para identificar e analisar jogos digitais para o apoio ao ensino-aprendizado, tendo como enfoque o conteúdo de pensamento computacional. Neste segundo caso, a metodologia também foi dividida em etapas, sendo elas, a seleção, categorização, análise e comparação entre jogos digitais.

Brito Junior e Aguiar (2014) afirmam que apesar de existirem diversas propostas na literatura que buscam definir uma forma adequada de selecionar e avaliar softwares educacionais, não existe um consenso sobre qual a melhor abordagem. Neste sentido, o presente artigo define um método para seleção de softwares de apoio ao ensinoaprendizagem de disciplinas de programação utilizando critérios qualitativos e quantitativos para avaliação. Desta forma, esta pesquisa soma-se aos esforços de Barbosa e Costa (2017) e Costa et al. (2015), ao propor um método para seleção de softwares e diferencia-se ao utilizar critérios de avaliação que consideram aspectos ergonômicos (relacionados à interação entre o homem e a máquina) e pedagógicos.

Dada a escolha de critérios e a definição de etapas genéricas, acredita-se que o método possa ser aplicado na seleção de softwares de apoio ao ensino-aprendizado de disciplinas de qualquer área de conhecimento. No entanto, considerando a motivação para definição da proposta e a necessidade de validação desta pesquisa, este artigo apresenta o estudo de caso que considera o uso do método focado na disciplina de programação de computadores I.

\section{Método de Seleção}

O método aplicado nesta pesquisa é fundamentado na análise de critérios qualitativos e quantitativos. Desta forma, busca-se avaliar e classificar os melhores softwares que poderão ser aplicados dentro do ambiente educacional como forma de auxílio para alunos e professores durante o processo de ensino-aprendizagem. Esta análise faz-se necessária para validar os resultados obtidos durante a pesquisa. Inicialmente, definiram-se as cinco principais etapas do método que são apresentadas na Figura 1.

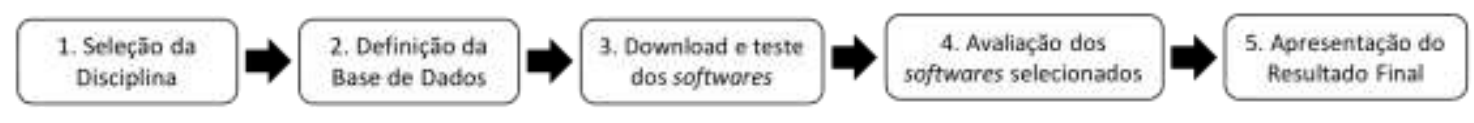

Figura 1. Etapas do Método de Seleção

$\mathrm{Na}$ etapa 1 é selecionada a disciplina, sua ementa, a fim de definir as palavraschave da busca baseadas na ementa da disciplina e no diálogo com o docente responsável em conduzir o curso. Na etapa seguinte é definida a base de dados de software que será explorada para a localização das possíveis soluções. Ainda na etapa 2 são utilizadas as palavras-chave de busca como entrada na base de dados escolhida. 
Deste modo, é realizado o levantamento dos possíveis softwares a serem adotados como ferramenta de ensino e aprendizagem. Na etapa 3, os softwares são baixados e testados para a criação de um ranking que lista em ordem de relevância os cinco (5) softwares mais indicados para uso. Os testes realizados nesta etapa devem levar em consideração os aplicativos que atendam os seguintes critérios de inclusão: (i) retornam com mais frequência quando aplicados as palavras-chave de busca adotadas na pesquisa; (ii) possuem as melhores avaliações de acordo com aquelas feitas por usuários que já usaram os aplicativos em questão; (iii) apresentam o maior número de instalações ativas; (iv) sejam direcionados para a área acadêmica; e que (v) sejam gratuitos. Além dos testes de uso, os softwares também são avaliados em relação ao feedback dos usuários em comentários. Já na etapa 4 , os cinco aplicativos que foram selecionados são instalados nos aparelhos celulares de onze profissionais da área sob investigação, que por sua vez são responsáveis em preencher um formulário eletrônico de avaliação composto por perguntas relacionadas às características ergonômicas e pedagógicas discutidas por Pereira et al. (2016). Deste modo, o método propõe de forma geral que a avaliação leve em consideração perguntas que discutam: (i) eficiência; (ii) interface gráfica; (iii) conteúdo; (iv) ajuda ao usuário; (v) controle do usuário; (vi) exercícios; (vii) instruções de uso; (viii) aspectos motivacionais; (ix) aprendizado; (x) compartilhamento entre usuários; e (xi) feedback positivo. Por fim, na etapa 5, os avaliadores apresentam o resultado final, acompanhado de um relatório quantitativo e qualitativo sobre as ferramentas selecionadas.

\section{Estudo de Caso}

Para validação do método proposto, realizou-se um estudo de caso a fim de selecionar um software gratuito de suporte ao ensino e aprendizagem da disciplina de Programação de Computadores I que considerasse aspectos ergonômicos e pedagógicos. A escolha se justifica pelo elevado índice de reprovações nesta disciplina, que de acordo com survey realizado por Viana et al. (2019), chega a uma média de cerca de 30\% da turma.

$\mathrm{Na}$ etapa 1 foram definidas as palavras-chave de busca, considerando a ementa da disciplina em questão que aborda os conceitos de programação orientada a objetos, utilizando a linguagem de programação Java. A Tabela 1 apresenta as palavras definidas juntamente com o professor da disciplina e suas respectivas justificativas. Ressalta-se que as palavras escolhidas nesta etapa, também foram combinadas durante o processo de busca.

Tabela 1. Palavras-chave de busca com suas respectivas justificativas

\begin{tabular}{|l|l|}
\hline Palavra-chave & Justificativa \\
\hline Java & $\begin{array}{l}\text { Verificar as possibilidades que a loja de aplicativos retornaria } \\
\text { com uma busca abrangente. }\end{array}$ \\
\hline Lógica de programação & $\begin{array}{l}\text { Obter aplicativos voltados ao ensino de lógica de programação } \\
\text { utilizando a linguagem Java. }\end{array}$ \\
\hline Aprenda Java & Obter aplicativos voltados a aprendizagem da linguagem Java. \\
\hline Ensino de Java & Obter aplicativos voltados ao ensino da linguagem Java. \\
\hline $\begin{array}{l}\text { Aprender lógica } \\
\text { programação com Java }\end{array}$ & $\begin{array}{l}\text { Obter aplicativos voltados ao ensino da linguagem Java que } \\
\text { fossem comuns as palavras-chave anteriores. }\end{array}$ \\
\hline
\end{tabular}


Na etapa 2 foi selecionada a loja virtual Google Play ${ }^{1}$ para busca e download dos softwares investigados. Durante esta etapa, as palavras-chave de busca foram concatenadas para garantir a maior abrangência dos resultados retornados pelo banco de dados. Durante as concatenações foi utilizado o caractere da soma (+) para juntar as palavras digitadas, por exemplo, "aprenda Java + Ensino de Java" ou "lógica de programação + Java". Após a realização da combinação das palavras, os aplicativos que mais se repetiram durante a busca foram os selecionados para a avaliação.

$\mathrm{Na}$ etapa 3 foi elaborada a lista de softwares voltados ao ensino e aprendizagem de Java, que foram retornados pela busca na loja virtual. Nesta etapa, cada resultado de busca retornou em média 250 aplicativos, dos quais os 100 primeiros foram selecionados para avaliação de acordo com a ordem de pontuação. Posteriormente, os cinco aplicativos com maiores notas foram selecionados e instalados nos celulares dos pesquisadores. Tais aplicativos são apresentados na Tabela 2, assim como, a nota e colocação no ranking gerado a partir dos critérios estabelecidos.

Tabela 2. Aplicativos selecionados com base nas notas obtidas

\begin{tabular}{|l|c|c|c|c|c|}
\hline \multicolumn{1}{|c|}{ Nome } & G & E & AC & DA & NF \\
\hline Aprenda Java & $\operatorname{Sim}$ & 4,4 & $\operatorname{Sim}$ & 50.000 & 5 \\
\hline Java Tutorial - offline & $\operatorname{Sim}$ & - & $\operatorname{Sim}$ & 10.000 & 5 \\
\hline Java Tutorial & $\operatorname{Sim}$ & 4.4 & $\operatorname{Sim}$ & 50.000 & 5 \\
\hline Learn Java & $\operatorname{Sim}$ & 4,6 & $\operatorname{Sim}$ & 1.000 .000 & 8 \\
\hline Java Solo & $\operatorname{Sim}$ & 4,1 & $\operatorname{Sim}$ & 5.000 & 4 \\
\hline
\end{tabular}

Gratuidade (G): Sim (2 pts); Não (0 pt). / Número de Estrelas (E): >= 4,5 (2 pts); < 4,5 (0 pt). / Voltado para Área Acadêmica (AC): Sim (2 pts); Não (0 pt). / Número de Downloads Ativos (DA): >= 500.000 (2 pts); < 500.000 E > 10.000 (1 pt); < 10.000 (0 pt). / Nota Final (NF): G + E + AC + DA (máximo 8 pts).

Já na etapa 4, os cinco aplicativos selecionados foram instalados nos aparelhos celulares dos onze profissionais que avaliaram. Tais profissionais são funcionários de uma empresa de tecnologia da informação e especialistas do Laboratório de Programação Extrema da Universidade Federal do Pará. Durante a avaliação, foram levadas em consideração as características ergonômicas e pedagógicas definidas no método apresentado neste trabalho. Por fim, na etapa 5, após avaliação dos aplicativos, os especialistas apresentaram os resultados que estão organizados na próxima seção.

\section{Resultados}

Os resultados referentes as 6 características ergonômicas são apresentadas na Tabela 3, enquanto que, os resultados sobre as 5 características pedagógicas estão dispostos na Tabela 4. Cada avaliador respondeu os formulários atribuindo sua resposta de acordo com a escala de Likert, isto é, CC - Concordo completamente (4 pts), C - Concordo (3 pts), D - Discordo (2 pts) e DC - Discordo completamente (1 pt). Considerando os 11 avaliadores, a nota máxima para cada característica poderia ser 44 pontos.

\footnotetext{
${ }^{1}$ Google Play é a loja de aplicativos da Google que concentra softwares desenvolvidos por empresas ou por programadores independentes.
} 
Considerando a análise ergonômica, é possível observar que os aplicativos: Aprenda Java, Java Tutorial offline, Java Tutorial e Learn Java, tiveram notas acima de 32 pontos no quesito eficiência; o aplicativo Java Solo foi o pior avaliado, obtendo nota 26. Em relação ao quesito interface agradável, os aplicativos Learn Java e Java Tutorial offline foram os únicos que obtiveram notas acima de 29, sendo os aplicativos que mais agradaram na questão visual. Em relação à organização dos conteúdos, todos os cinco aplicativos conseguiram obter notas superiores a 30, isto é, notas entre 32 e 38. Apesar dos aplicativos terem obtido notas baixas, em outros quesitos, estes, conseguem transmitir o conteúdo de forma clara e objetiva na opinião dos especialistas/avaliadores.

Em relação a ajuda disponibilizada, o único que obteve nota acima de 30 foi o aplicativo Learn Java, o qual alcançou a nota 35. Os demais aplicativos não disponibilizam esse tipo de suporte para o usuário, retornando, deste modo, a nota 0 (sem avaliação). Já no quesito controle do usuário, o aplicativo Learn Java, obteve a maior nota, 33, sendo o único dos cinco que é divido em módulos que necessita da finalização de determinada atividade para o desbloqueio do próximo módulo. Por fím, em relação ao quesito exercícios, constatou-se, que o aplicativo Java tutorial é o único dos cinco aplicativos que não apresenta exercício para que o usuário possa testar o conhecimento que foi adquirido, durante o estudo, obtendo a pior nota neste quesito.

Tabela 3. Avaliação dos aplicativos com base nos critérios ergonômicos

\begin{tabular}{|l|c|c|c|c|c|}
\hline Critérios/Ferramentas & $\begin{array}{c}\text { Aprenda } \\
\text { Java }\end{array}$ & $\begin{array}{c}\text { Java Tutorial } \\
\text { offline }\end{array}$ & $\begin{array}{c}\text { Java } \\
\text { Tutorial }\end{array}$ & $\begin{array}{c}\text { Learn } \\
\text { Java }\end{array}$ & $\begin{array}{c}\text { Java } \\
\text { Solo }\end{array}$ \\
\hline Eficiente & $33 \mathrm{pts}$ & $35 \mathrm{pts}$ & $33 \mathrm{pts}$ & $34 \mathrm{pts}$ & $26 \mathrm{pts}$ \\
\hline Interface agradável & $28 \mathrm{pts}$ & $30 \mathrm{pts}$ & $29 \mathrm{pts}$ & $37 \mathrm{pts}$ & $27 \mathrm{pts}$ \\
\hline Conteúdo claro e objetivo & $37 \mathrm{pts}$ & $38 \mathrm{pts}$ & $32 \mathrm{pts}$ & $37 \mathrm{pts}$ & $33 \mathrm{pts}$ \\
\hline Ajuda disponibilizada & $21 \mathrm{pts}$ & $28 \mathrm{pts}$ & $25 \mathrm{pts}$ & $35 \mathrm{pts}$ & $25 \mathrm{pts}$ \\
\hline Controle do usuário & $30 \mathrm{pts}$ & $32 \mathrm{pts}$ & $28 \mathrm{pts}$ & $33 \mathrm{pts}$ & $29 \mathrm{pts}$ \\
\hline Exercícios & $31 \mathrm{pts}$ & $38 \mathrm{pts}$ & $26 \mathrm{pts}$ & $32 \mathrm{pts}$ & $35 \mathrm{pts}$ \\
\hline Total Geral & $\mathbf{1 8 0} \mathbf{~ p t s}$ & $\mathbf{2 0 1} \mathbf{p t s}$ & $\mathbf{1 7 3} \mathbf{~ p t s}$ & $\mathbf{2 0 8} \mathbf{p t s}$ & $\mathbf{1 7 5} \mathbf{p t s}$ \\
\hline $\begin{array}{l}\text { Porcentagem em relação } \\
\text { à pontuação }\end{array}$ & $\mathbf{1 9 , 2 1 \%}$ & $\mathbf{2 1 , 4 5 \%}$ & $\mathbf{1 8 , 4 6 \%}$ & $\mathbf{2 2 , 2 0 \%}$ & $\mathbf{1 8 , 6 8 \%}$ \\
\hline
\end{tabular}

Com base nos critérios ergonômicos, é possível observar que os aplicativos que mais se adequaram a proposta de utilização para ensino e aprendizagem foram: em primeiro lugar, o Learn Java, obtendo 22,2\%; seguido pelo aplicativo Java Tutorial offline, com 21,45\%; Aprenda Java, obtendo 19,21\%; Java Solo, alcançando 18,68\% e, por fim em quinto lugar, Java Tutorial, obtendo 18,46\%.

Na Tabela 4, apresenta-se a avaliação com relação às características pedagógicas. Segundo as avaliações feitas pelos profissionais, observa-se que: as instruções e 
exemplos estão pedagogicamente de acordo com o proposto pelo conteúdo e aprendizado, no que se refere a absorção de conteúdo pelo usuário. Os cinco aplicativos apresentaram notas altas nestes quesitos avaliados. No quesito aspectos motivacionais, no qual se verifica a possibilidade de o usuário ter motivação para continuar usando o aplicativo, os aplicativos Learn Java e Java Tutorial offline obtiveram as maiores notas, 31 pts e 37 pts, respectivamente. Quanto ao feedback para o usuário, os aplicativos Aprenda Java e Learn Java ficaram com as melhores notas, 30 pts e 35 pts, respectivamente. Levando em consideração os critérios pedagógicos, concluiu-se que os aplicativos Learn Java e Java Tutorial offline, mantiveram as melhores avaliações.

Como observado nas Tabelas 3 e 4, os aplicativos foram classificados de acordo com a somatória total das suas notas. Cada tabela gerou uma porcentagem de classificação, que ao final foram somadas e encontrada a média de suas respectivas notas, sendo classificados da seguinte forma: Learn Java 22,71\%, Java tutorial offline 21,38\%, Aprenda Java 19,25\%, Java Tutorial 18,56\% e Java Solo 18,1\%.

Tabela 4. Avaliação dos aplicativos com base nos critérios pedagógicos

\begin{tabular}{|l|c|c|c|c|c|}
\hline \multicolumn{1}{|c|}{ Critérios/Ferramentas } & $\begin{array}{c}\text { Aprenda } \\
\text { Java }\end{array}$ & $\begin{array}{c}\text { Java Tutorial } \\
\text { offline }\end{array}$ & $\begin{array}{c}\text { Java } \\
\text { Tutorial }\end{array}$ & $\begin{array}{c}\text { Learn } \\
\text { Java }\end{array}$ & $\begin{array}{c}\text { Java } \\
\text { Solo }\end{array}$ \\
\hline $\begin{array}{l}\text { As instruções e exemplos estão } \\
\text { pedagogicamente de acordo } \\
\text { com o proposto pelo conteúdo. }\end{array}$ & $35 \mathrm{pts}$ & $37 \mathrm{pts}$ & $37 \mathrm{pts}$ & $37 \mathrm{pts}$ & $34 \mathrm{pts}$ \\
\hline Aspectos motivacionais & $28 \mathrm{pts}$ & $31 \mathrm{pts}$ & $26 \mathrm{pts}$ & $37 \mathrm{pts}$ & $23 \mathrm{pts}$ \\
\hline Aprendizado & $31 \mathrm{pts}$ & $36 \mathrm{pts}$ & $34 \mathrm{pts}$ & $36 \mathrm{pts}$ & $32 \mathrm{pts}$ \\
\hline $\begin{array}{l}\text { Indicaria o aplicativo para } \\
\text { outras pessoas? }\end{array}$ & $28 \mathrm{pts}$ & $35 \mathrm{pts}$ & $26 \mathrm{pts}$ & $38 \mathrm{pts}$ & $26 \mathrm{pts}$ \\
\hline Feedback & $30 \mathrm{pts}$ & $29 \mathrm{pts}$ & $24 \mathrm{pts}$ & $35 \mathrm{pts}$ & $23 \mathrm{pts}$ \\
\hline Total Geral & $\mathbf{1 5 2} \mathbf{p t s}$ & $\mathbf{1 6 8} \mathbf{p t s}$ & $\mathbf{1 4 7} \mathbf{p t s}$ & $\mathbf{1 8 3} \mathbf{p t s}$ & $\mathbf{1 3 8} \mathbf{p t s}$ \\
\hline $\begin{array}{l}\text { Porcentagem em relação à } \\
\text { pontuação }\end{array}$ & $\mathbf{1 9 , 2 9 \%}$ & $\mathbf{2 1 , 3 2 \%}$ & $\mathbf{1 8 , 6 6 \%}$ & $\mathbf{2 3 , 2 2 \%}$ & $\mathbf{1 7 , 5 1 \%}$ \\
\hline
\end{tabular}

Como os aplicativos Learn Java e Java tutorial offline ficaram em primeiro e segundo lugar respectivamente, foram classificados como os dois mais adequados, visto que houver um empate técnico, para esta pesquisa, validando, assim, que o método está apto para selecionar os aplicativos que podem apoiar o ensino e aprendizagem de uma disciplina. Seguindo os passos propostos pela pesquisa, este trabalho, também, apresenta como possibilidade a escolha de softwares que possam atender aos critérios ergonômicos e pedagógicos necessários para o ensino e aprendizagem de disciplinas de outras áreas, carecendo de estudos de caso específicos para tal validação.

Os resultados demonstraram que o aplicativo Learn Java conseguiu sanar com mais precisão as questões que foram propostas na pesquisa, tornando-se, com isso, o mais propício de ser utilizado dentro de um ambiente educacional. $\mathrm{O}$ modelo proposto por este aplicativo faz com que os usuários precisem seguir os módulos de acordo com 
seu desemprenho. Sua interface é mais agradável, porém precisa ser realizado o login para ter acesso ao aplicativo. Já o aplicativo Java tutorial offline, consegue sanar as necessidades, porém não segue um roteiro de aprendizagem, visto que disponibiliza todo o conteúdo para ser consultado a qualquer momento, ou seja, o usuário decide quais tutoriais deseja seguir.

\section{Considerações Finais}

Este trabalho teve como objetivo apresentar um método para seleção de softwares de suporte ao ensino-aprendizagem de alunos de disciplinas de programação. Para validar o método, foi realizado um estudo de caso com a seleção de cinco aplicativos relacionados à disciplina de Programação de Computadores I. A partir das análises que foram aplicadas nesta pesquisa, foi possível constatar, que o método proposto para selecionar os aplicativos, está apto para ser aplicado na seleção de determinado software no âmbito do processo de ensino e aprendizagem.

Foi observado, porém, que os aplicativos voltados para o ensino da linguagem Java não seguem um padrão pedagógico em seu desenvolvimento; com isso, levantou-se a seguinte questão "os aplicativos voltados para o ensino e aprendizagem são avaliados, tanto por especialistas das suas respectivas áreas, quanto por especialista da área educacional?". Certamente, o desenvolvimento de softwares voltados para área educacional precisa de uma atenção maior, pois esses serão aplicados no ensino aprendizagem e, sem dúvidas, precisam ser cuidadosamente planejados, pois as informações propagadas devem ser de alta qualidade. Neste sentido, por utilizar critérios objetivos e genéricos, acredita-se que o método proposto pode ser utilizado para seleção e avaliação de softwares educacionais de outras áreas, além da disciplina de programação no qual foi validado.

Como trabalho futuro, esta pesquisa propõe o desenvolvimento de um software que sistematize a avaliação das características ergonômicas e pedagógicas que foram abordadas durante a pesquisa. Durante o desenvolvimento pela equipe de tecnologia, esse software deverá ser acompanhado pelos especialistas da área pedagógicas. Também, os autores pretendem disponibilizar o método para que outros aplicativos de outras áreas possam desenvolver seus respectivos aplicativos.

\section{Referências}

Barbosa, A. e Costa, F. (2017) "Seleção e avaliação de ferramentas para a promoção do Pensamento Computacional”, https://repositorio.ufpb.br/jspui/handle/123456789/44381.

Barcelos, R., Tarouco, L. e Berch, M. (2009). O Uso de Mobile Learning no Ensino de Algoritmos. Em Revista Novas Tecnologias na Educação, v. 7, n. 2.

Bittencourt, R., Rocha, A., Santana, B., Santana, C., Carneiro, D., Borges, G., Chalegre, H., Silva, J., Santos, J., Silva, L. e Andrade, P. (2013). "Aprendizagem de Programação Através de Ambientes Lúdicos em um Curso de Engenharia de Computação: Uma Primeira Incursão”. Em: Workshop sobre Educação em Computação (WEI), Maceió.

Brito Júnior, O. e Aguiar, Y. (2014). “Análise de abordagens objetivas para avaliação de softwares educativos". Em: Simpósio Brasileiro de Fatores Humanos em Sistemas Computacionais (IHC), Foz do Iguaçu. 
Carlet, E. e Czyzewski, A. (2016). O Uso da Lousa Digital como Recurso Pedagógico no Processo de Ensino Aprendizagem na Educação Especial. Em Cadernos PDE, vol. I.

Costa, S., Souza, S., Mendes, C., Obregon, R., Da Silva, L., Moreira, E. e Ferreira, J. (2015). "Um estudo exploratório dos games para introdução ao pensamento computacional". Em: Congresso Nacional de Ambientes Hipermídia para Aprendizagem (CONAHPA), São Luís.

DATAFOLHA. (2014) "43 Milhões de Brasileiros Acessam Internet por Dispositivos Móveis", http://datafolha.folha.uol.com.br/mercado/2014/01/1400618-43-milhoes-debrasileiros-acessam-internet-por-dispositivos-moveis.shtml.

Gouvêa, M. e Nakamoto, P. (2015). "Avaliação de Software Educacional: Uma Oportunidade de Reflexão da Educação na Sociedade do Conhecimento". Em: Congresso Internacional Trabalho Docente e Processos Educativos, Uberaba.

Lima, M., Lima, L., Neto, C. e Castro Filho, J. (2016). "Experiência de Aprendizagem Móvel: o Uso do WhatsApp no Ensino Fundamental". Em: Congresso Brasileiro de Informática na Educação (CBIE), Uberlândia.

Martínez-Rodríguez, R., Alvarez-Xochihua, O., Victoria, O., Arámburo, A. and Fraga, J. (2019). Use of Machine Learning to Measure the Influence of Behavioral and Personality Factors on Academic Performance of Higher Education Students. In IEEE Latin America Transactions, v. 17, n. 04, pages 633-641.

Nunes, R. e Santos, I. (2018). “A Importância da Avaliação Ergonômico-Pedagógica de Aplicativos Educacionais e os Desafios Encontrados no Campo da Aprendizagem Móvel”. Em: Congresso Brasileiro De Informática Na Educação (CBIE), páginas 41-50.

Nunes, F., Herpich, F., Paschoal, L., Tarouco, L. e De Lima, J. (2017). "Mundos Virtuais na Prática Docente: uma Visão sobre os Desafios e Benefícios". Em: Workshop sobre Educação em Computação (WEI), Salvador, páginas 845-854.

Oliveira, T., Bitencourt, R. e Monteiro, W. (2016). "Experiências com Gamificação no Ensino de Computação para Jovens e Adultos no Sertão Pernambucano”. Em: Simpósio Brasileiro de Games e Entretenimento Digital (SBAGAMES), São Paulo.

Oliveira, N., Farias, F., Moura, D. e Anjos, J. (2017). “Aprendendo Lógica Computacional Usando o Aplicativo AlgoFácil”. Em: Congresso de Tecnologias e Desenvolvimento na Amazônia (CTDA), Cametá.

Pereira, W., Silva, R., Filho, R., Silva, W., Aguiar, Y. e Dantas, V. (2016). Avaliação de Software Educativo: Análise de Abordagens para Definição de Diretrizes. Em Nuevas Ideas en Informática Educativa, v. 12, páginas 557-562.

Silveira, R. e Alcantara, S. (2014). "Relato da Experiência do Trabalho com Jogos Manuais de Raciocínio Lógico como Reforço para as Disciplinas de Algoritmos e Linguagem de Programação". Em: Simpósio Brasileiro de Informática na Educação (SBIE), Santa Maria, páginas 983-987.

Twining, P., Raffaghelli, J., Albion, P. and Knezek, D. (2013). Moving Education into the Digital Age: The contribution of Teachers' Professional Development. Journal of Computer Assisted E-Learning, 29 (5), pages 426-437.

Viana, G., Lopes, A., Portela, C. e Oliveira, S. (2019). "Um Survey sobre a Aprendizagem de Programação no Curso de Sistemas de Informação”. Em: Workshop Sobre Educação em Computação (WEI), Belém, páginas 161-175. 